\title{
Fast and Robust Road Sign Detection in Driver Assistance Systems
}

\author{
Tao Zhang · Jie Zou · Wenjing Jia
}

Received: date / Accepted: date

\begin{abstract}
Road sign detection plays a critical role in automatic driver assistance systems. Road signs possess a number of unique visual qualities in images due to their specific colors and symmetric shapes. In this paper, road signs are detected by a two-level hierarchical framework that considers both color and shape of the signs. To address the problem of low image contrast, we propose a new color segmentation algorithm based on color visual saliency, which uses the ratios of enhanced and normalized color values to capture color information. To improve computation efficiency and reduce false alarm rate, we modify the fast radial symmetry transform (RST) algorithm, and propose to use an edge pairwise voting scheme to group feature points based on their underlying symmetry in the candidate regions. Experimental results on several benchmarking datasets demonstrate the superiority of our method over the state-of-the-arts on both efficiency and robustness.
\end{abstract}

Keywords Road sign detection · Visual saliency · Normalized RGB colors · Improved radial symmetry transform $($ IRST) $\cdot$ Real-time applications

\section{Introduction}

In recent years, traffic accidents have caused great economic loss, injuries and deaths. Furthermore, many older drivers face impaired visual functioning cataract and the related visual impairment is highly prevalent. There is an increasing number of in-vehicle entertainment systems which dramatically increases the chance of car accidents as a result of driver distraction. As an essential task for regulating traffic and guiding and warning drivers and pedestrians, automatic detection of road signs provides information of the current state of the road, forbidden maneuvers, the right-of-way, etc, and has received more and more attentions $[17,16]$.

\section{T. Zhang}

Key Laboratory of Advanced Process Control for Light Industry (Ministry of Education)

Jiangnan University

E-mail: taozhang@jiangnan.edu.cn

\section{J. Zou( $(\bowtie)$}

Department of Computer Science and Technology

Wuhan Technology and Business University, the corresponding author of this paper

E-mail: qvbso0724@163.com

W. Jia

Faculty of Engineering and Information Technology

University of Technology Sydney, Australia

E-mail: Wenjing.Jia@uts.edu.au 


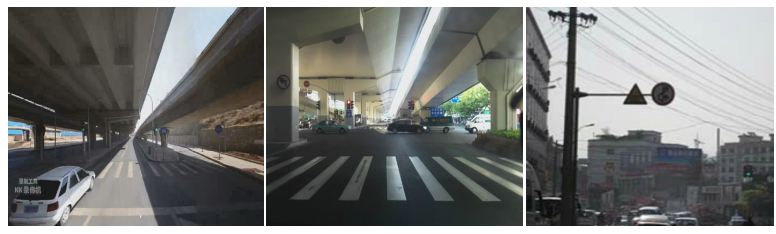

(a) Low contrast images

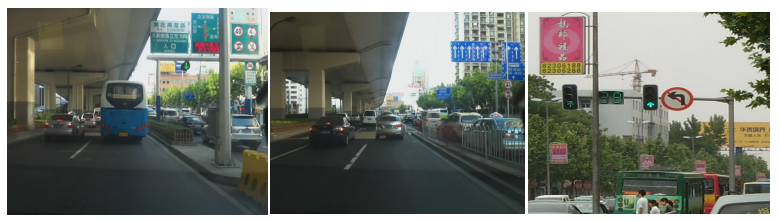

(b) Complex background

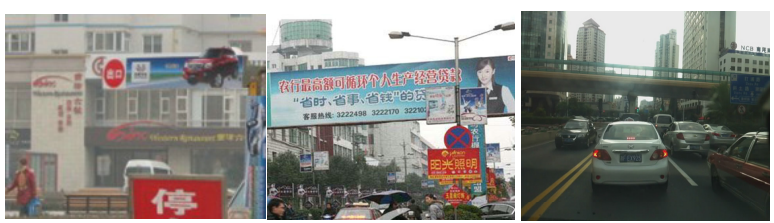

(c) Other objects similar to road signs

Fig. 1 Examples of some difficult cases.

Detecting road signs is the most critical step of a road sign recognition system. However, there exist a number of difficulties which make it still a challenging task, such as complex background and illumination, shape variations caused by translation and rotation, low resolution, noisy and obscured signs, differences of the same signs, other objects with the same color and texture, etc, (see Fig. 1 for some examples of difficult cases). The difficulty in exploiting sufficient information from road sign images makes the detection problem even more challenging. Furthermore, the computational complexity increases rapidly to reach higher detection rates. In order to implement such practical road sign recognition systems, developing technologies used for automated detection of road signs has been an important issue for researchers in computer vision field, where many researchers have achieved significant progress [36].

In general, the main cause of car-accidents are speed limit exceeding and driver distractions. Therefore, to help keep the speed below the limit and to avoid distraction while driving are the two main targets in this work which focuses on road sign detection for driving assistance. Thus, our system will alert drivers, but will not actuate to control the vehicle. We define drivable road regions as a connected region in front of the vehicle on the road surface where the vehicle can pass safely taking no account of shadows, markings or traffic regulations. A desirable detection algorithm should be able to extract all drivable road regions from a road image regardless of disturbance such as shadows, lane markings or uneven lighting. In this paper, the main task of our work is to design a fast method for detecting road signs in a sequence of images. Our goal is to construct a robust framework that can detect road signs of any countries with any of the four shapes (circle, triangle, rectangle and octagon).

Up to now, many solutions have been proposed to address the difficult detection problems. These approaches can be roughly classified into shape-based [19,11,30,29], color-based [39,27, $10,15]$ and supervised-training based approaches [26,2,28,23].

The shape-based approaches use the geometric structure feature of road signs for detection. In [19], Andrey et al. proposed an algorithm for detecting and recognizing restricting, warning, and information road signs, which was fast enough for real-time processing. However, it only considered particular signs by using a simple combination of color and shape feature, and therefore its false-positive rate was high. In [11], Escalera et al. proposed to use the deformable model scheme to detect road signs. It was robust in uncontrolled lighting conditions with occlusions, variations in shape, size and color but had a problem of high computational cost. Parada-Loira et al. [31] proposed a two-stage sign detection system. This system demonstrated a very good performance for multi-class traffic sign detection. However, it relied on local contour patterns, 


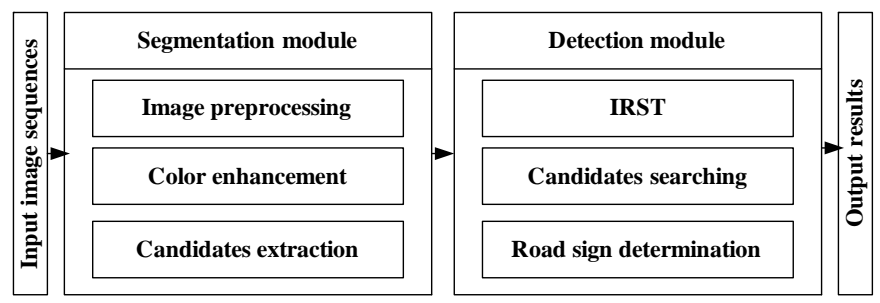

Fig. 2 The framework of the proposed method

while the complete contour of a road sign region can be difficult to achieve. In [30], Paclik et al. proposed a new kernel rule and used the Laplace probability density to classify road signs. However, it mainly focused on the recognition step and ignored the most important detection step, and therefore in real-life applications it did not perform very well. Later, in [29], Paclik et al. built road-sign classifiers using a trainable similarity measure, where the similarity was based on individual matches in a set of local image regions, but it had the problem of sample selection. Shape-based approaches use the geometric structure feature of road signs for detection. However, this kind of algorithm relied on local contour patterns, while the complete contour of a road sign region can be difficult to achieve. Color-based approaches are mainly based on color features. This kind of algorithm often failed when background was complex, especially in the cases where other objects were with the similar color and texture to road sign region. The supervised training-based approaches are mainly based on support vector machines (SVMs) and their performance can be easily affected by low image contrast.

In working with the color-based approaches, searching is mainly based on the color feature. In [4], Benallal and Meunier proposed a real-time color segmentation method for road signs. Their method only considered a certain type of specific signs by using their color features. When there were other objects with the color and texture similar to those of road sign regions, it failed to differentiate them. In [39], a fast and robust framework for incrementally detecting text on road signs from video was presented. This method became invalid when background was complex, especially in the cases where other objects were with the similar color and texture to road sign region. Nguwi and Kouzani [27] presented an automatic road sign recognition system capable of analyzing live images, detecting multiple road signs within images, and classifying the type of the detected road signs. However, it operated on HSI color space, which was not robust under different illuminations. A genetic algorithm and neural network were used to detect and recognize road sign respectively in [10]. The method required a lot of fine-tuned parameters and therefore was not adaptive to different environment, and also its computational cost was very high. Gomez et al. [15] described a general framework for detecting and tracking traffic and road signs from image sequences using only color information. This method is robust to different lighting conditions and partial occlusion, but is not suitable for real-time applications.

The supervised-training approaches are mainly based on support vector machines (SVMs). Maldonado-Bascn et al. [26] presented an automatic road-sign detection and recognition system based on SVMs. This method can achieve a high success rate and a very low false positive rate in the recognition stage. However, its performance can be easily affected by low image contrast. In [2], [35] and [36], the AdaBoost algorithm has been used for robust real-time traffic sign detection, tracking, and recognition. In order to achieve a high accuracy, lots of training samples were required, which can be difficult to obtain in practice. Escalera [12] introduced a novel system for multi-class classification of traffic signs based on error correcting output codes. The presented multi-classification system was robust when dealing with a large number of classes. However, it did not perform well for the cases with complex background, and also its computational cost was high. In [28], Overett et al. designed a system for creating object detectors which met the extreme demands of real-world traffic sign detection applications such as GPS map making and real-time in-car traffic sign detection. This method expanded their use of Haar features to include color information and has achieved a high accuracy rate. However, for some types of signs (such as the No Entry signs), its performance was not good. In [23], 
Liang et al. presented an efficient road sign detection method using supervised learning, which has achieved a high detection rate. However, for those mandatory road signs, its performance was not good.

Additionally, Timofte et al. [37] proposed a pipeline for efficiently detecting and recognizing traffic signs by combining 2D and 3D techniques. Khan et al. [20] proposed an automatic roadsign recognition method based on image segmentation and joint transform correlation with the integration of shape analysis. The proposed framework is invariant to translation, rotation, scale and partial occlusions. Although these methods have made an attempt to resolve the aforementioned problems, they have their own limitations and road sign detection is still an open challenge for computer vision community.

Until very recently, quite a number of papers have been published on road sign detection. In [8], a two-step road sign detection method was proposed, which was robust to many kinds of adverse situations including poor lighting condition, partial occlusion, rotation, etc. However, the robustness of the method to occlusions still needs to be improved. Ref. [5] introduced a system that can detect and recognize road signs. Their experiments were conducted on RGB images taken from both urban and non-urban roads at different times of the day. However, it did not mention the use of other methods for classification and recognition processes. [33] proposed a road surface traffic sign detection system by applying convolutional neural networks (CNNs). Extensive experiments have been conducted using their field-captured dataset, demonstrating outstanding recall and precision rate. However, the designed deep CNN models have too many layers and network parameters, which leads to a high processing time. To reduce this high processing time, [1] proposed a modification of SegNet, a deep fully convolutional neural network for image segmentation. Their work differs from previous work on adversarial sample crafting and defense against adversarial samples in a way that they are aggregating, applying and verifying the previous methods in the context of traffic signs. Ellahyani et al. [9] proposed a new method for road sign detection by employing both a random forest classifier and a shape based classification technique. The proposed detection method has achieved satisfactory results. [22] proposed a method to detect road signs with various aspect ratios by identifying corners and combining such elements. By verifying each step to maximize detection performance and by tracking the bottom corners, the corners at close range can be used. The proposed method is applied to the images acquired on the highway, and high detection performance and real-time operation can be confirmed. Gomes et al. [14] proposed the detection and recognition of speed limit signs based on a cascade of boosted classifiers working with Haar-like features. The recognition of the sign detected is achieved based on the optimum-path forest classifier (OPF), support vector machines (SVM), multilayer perceptron, k-nearest neighbor $(\mathrm{kNN})$, extreme learning machine, least mean squares, and least squares machine learning techniques.

To address the aforementioned difficulties and aim to develop an effective, practical algorithm that can handle a large variety of road signs at low computational cost, we propose a two-level hierarchical framework, as illustrated in Fig. 2. The input image sequence firstly goes through a segmentation module to obtain candidate regions which may contain road signs. These candidate regions are then identified as either a road sign or non-road sign by a detection module.

The main motivation of this paper is to present a fast and robust road traffic sign detection system. The main characteristics of our proposed system should include: 1) A hybrid region proposal method which takes into account the complementary information of color and edge. 2) A fast road sign location algorithm.

In the context of this framework, our main contributions in this paper are two-fold: 1) When generating candidate regions, we propose a novel color segmentation scheme based on visual saliency; 2) For sign recognition, instead of using the conventional shape-based features, we modify the fast Radial Symmetry Transform (RST) and propose an improved RST, named as IRST, which helps to detect road signs more efficiently and accurately.

The rest of the paper is organized as follows. Sect. 2 focuses on the related research works. Sects. 3 and 4 detail the two above mentioned modules of our proposed algorithm. Sect. 5 presents experimental results where the performance of our approach is compared with the state-of-the-art approaches. We conclude the paper in Sect. 6 . 


\section{System Overview and Related Work}

The approach proposed in this paper follows the major trend of existing works and uses the combination of multiple cues for road sign detection. In particular, aiming at a robust and fast system, we propose to detect road signs in a multi-stage process and build a two-level hierarchical framework, which includes a segmentation module and a detection module, as shown in Fig. 2.

In the segmentation phase, the system searches the input images for candidate regions of road signs using a simple but effective new method based on color visual saliency. In the detection phase, each extracted candidate region is further examined using a more complicated shapebased approach to determine whether it is a real road sign. We modify the fast RST algorithm and propose an improved RST so as to achieve higher efficiency while retrieving more targets. Experimental results demonstrate that our proposed method is not only robust against various illumination conditions but also appealing from the implementation point of view due to its simpleness and fast computation.

\subsection{Related Works Using Color Segmentation}

Image segmentation techniques have been widely used in various applications involving object detection. For road-sign detection, two main types of segmentation techniques have been used, i.e., 1) edge based approaches, and 2) color based approaches.

In the edge based approaches, searching mainly focuses on the contour of targets. Since most shape detectors involve computationally expensive operations, this may result in slow processing, especially for large images with cluttered background. As a comprising alternative, gradient based feature detection is less computationally expensive, but its performance can be very sensitive to noisy edges appearing in cluttered background.

In the color based approaches, the main idea is to use standard color spaces and develop a more exhaustive color study [7]. The authors in [4,6] applied thresholding directly on the RGB color values of pixels to segment regions of interest. Instead, most other researchers have chosen to operate on other color spaces which are less sensitive to illumination changes. The hue-saturation-intensity (HSI) color space has therefore become the most common choice [10, 11,26]. However, the HSI space cannot cover all the possible cases. The LUV and YUV spaces have also been used to segment road signs [27].

In order to overcome the problems arisen in complex environment, some researchers $[38,32$, 13,18 ] have considered color visual saliency techniques which are based on biologically motivated selective attention mechanism to imitate human-like early visual processing.

Inspired by these successes, in order to handle the cluttered background, we also consider color visual saliency and propose a novel method to use the color visual saliency of images to adapt to various illumination conditions. More details of this idea will be given in Section 3 .

\subsection{Related Works using Shape Analysis}

Using shape based features for road sign detection have been frequently reported in literature. For instance, in [10], the road signs were further verified through a shape analysis method embedded in genetic algorithm (GA). In [19], road signs were classified as a circle or triangle using background shape histograms. Note that, this method faces challenges of dealing with cluttered background. The authors in [34] proposed to discriminate local regions based on shape features to select candidate road signs. In [13], shape features extracted by a vision model "FOSTS" were used to classify road signs.

The symmetry characteristic of objects, as an intrinsic phenomenon in the world around us, has attracted researchers' attentions and symmetry detection has been used for numerous applications. This can be computational intensive, until Loy and Zelinsky [25] proposed the fast Radial Symmetry Transform (RST) for detecting points of interest. Its low computational complexity and fast speed makes this method well-suited for real-time vision applications. Following 
this work, Loy and Barnes [24] introduced a novel shape-based technique and used it to detect road signs in images. The method is invariant to in-plane rotation, being able to detect signs viewed at any orientation, and returns the location and size of the shape detected, and it has been shown to effectively detect the circles on Australian speed signs.

\section{The Segmentation Module}

The key goal of this module is to obtain candidate road sign regions for further verification.

In existing research, many researchers have used saliency-map models to simulate the visual attention mechanism (e.g. [13,18,32,38]). In [13], colour appearance model CIECAM97 has been applied to extract colour information. However, it does not perform well at detecting targets in low contrast scenes. In [18], multiscale image features are combined into a single topographical saliency map. A dynamical neural network then selects attended locations in order of decreasing saliency. Despite its simple architecture and feed-forward feature-extraction mechanisms, the model is capable of strong performance with complex natural scenes. However, it will fail at detecting targets salient for unimplemented feature types (e.g., T junctions or line terminators). Sang-Jae [32] proposed a new saliency map model with an adaptive masking method, which was based on three pre-processed channels of color and edge with the adaptive masking operator. However, it can not distinguish these objects similar to road signs. Woong-Jae [38] considered the color opponents and their edge information with center surrounding difference and normalization as a pre-processing, which is effective to intensify the sign board color characteristics as well as reducing background noise influence. In real environment, the proposed model can successfully localizes a salient area and detects the road traffic sign regions. However, its performance drops greatly in complex scenes. This kind of models are based on bottom-up, scene-based properties. It reduces computation by a selection on the basis of pre-attentively computed simple features and addresses some problems with the integration of different feature dimensions into a spacerelated map.

Inspired by above works, we try to find the salient areas according to various stimuli in a static color image. Since the colors of road sign boards typically own good contrast level against their ambient environment (so as to readily attract attentions from road users), we propose to use a novel visual saliency method to segment input images into candidate regions. In this method, road sign candidate regions are filtered by color ratios of enhanced, normalized color values. This is illustrated in Fig. 3 with details provided next.

Note that, several researchers have suggested using the HSV and YCbCr color space instead of the RGB color space [10,11]. We have compared the results obtained on HSV, YCbCr, RGB and a normalized RGB space and found that our color ratios of color-enhanced, normalized RGB worked the best.

For any given pixel of the input color image, let us denote the values of its red, green and blue channel as $R, G$ and $B$ respectively. The corresponding normalized values, denoted as $r, g$ and $b$, and yellow $(y)$, are defined as:

$$
\begin{aligned}
& r=\max \left(0, R-\frac{G+B}{2}\right) \\
& g=\max \left(0, G-\frac{R+B}{2}\right) \\
& b=\max \left(0, B-\frac{R+G}{2}\right) \\
& y=\max \left(0, \frac{R+G}{2}-B\right),
\end{aligned}
$$

where the function $\max (X, Y)$ returns the larger value between $X$ and $Y$.

Then, in order to depict the color characteristics of road signs of different colors, we propose a novel color enhancement scheme based on visual saliency using color ratios, as defined in Eq. 1, 2 and 3. In particular, we have targeted three types of road signs, i.e., warning signs (generally with yellow color), prohibitory signs (generally with red color), and guiding signs (generally with 


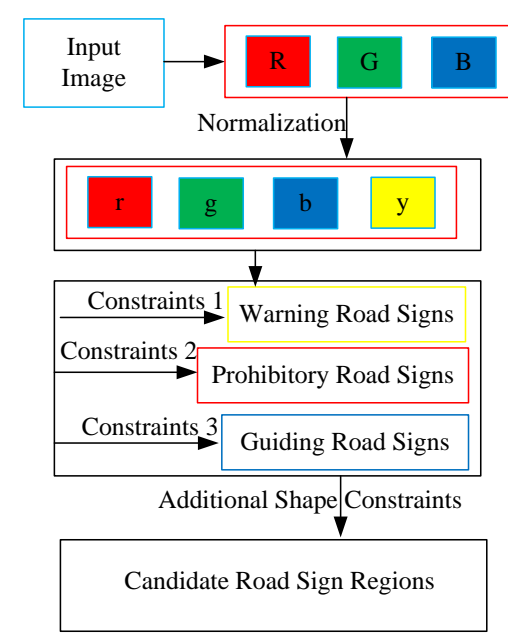

Fig. 3 The flowchart of our candidate region generation method based on color visual saliency.
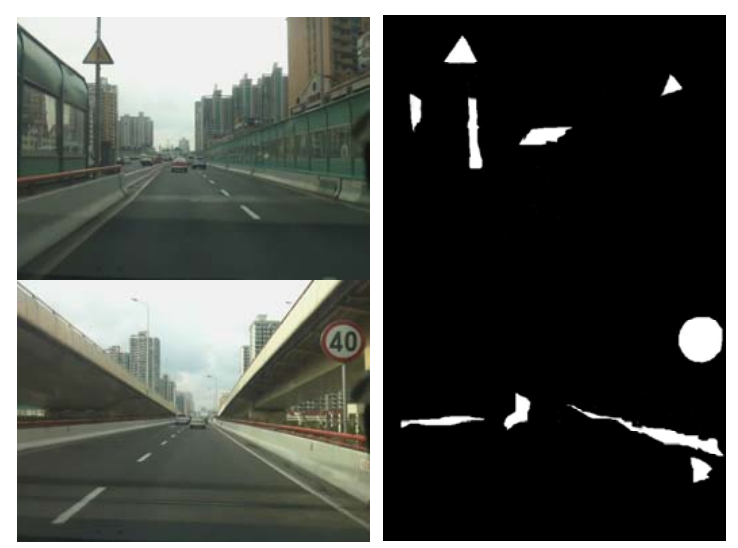

Fig. 4 Segmentation results of the examples (top) and a prohibitory sign (bottom). Left: original images; right: binary maps.

blue color). For other signs (e.g. the guiding signs on highways which are with white letters on green background), a similar set of constraints can be collected, making it very easy to expand to deal with other ranges of signs.

For pixels belonging to warning signs (generally with yellow color), their $g, b$, and $y$ values conform to the following constraints:

$$
\frac{y}{y+g}>\alpha_{w a r n} \& \frac{y}{y+b}>\beta_{w a r n} \& \frac{g}{g+b}>\lambda_{w a r n}
$$

For pixels belonging to prohibitory signs (generally with red color), their $r, g, b$ values conform to the following constraints:

$$
\frac{r}{r+g}>\alpha_{r e d} \& \frac{r}{r+b}>\beta_{r e d} \& \frac{g}{g+b}>\lambda_{r e d}
$$

Whereas for pixels belonging to guiding signs (generally with blue color), their $g, b, y$ values conform to the following constraints:

$$
\frac{b}{b+g}>\alpha_{\text {blue }} \& \frac{b}{b+y}>\beta_{\text {blue }} \& \frac{g}{g+b}>\lambda_{\text {blue }} \text {. }
$$

Here, $\alpha_{\text {warn }}, \alpha_{\text {red }}, \alpha_{\text {blue }}, \beta_{\text {warn }}, \beta_{\text {red }}, \beta_{\text {blue }}, \lambda_{\text {warn }}, \lambda_{\text {red }}$ and $\lambda_{\text {blue }}$ are all constants. In this paper, we use a naive Bayesian model to statistically learn the optimal values by sampling the corresponding values of the color-normalized images of typical signs using Eq. 1. 
The above constraints are applied to the image pixel by pixel and result in a binary image where non-zero pixels form candidates of road signs. Each class can produce its own binary image, and then all classes of binary images are combined into a single binary image.

In order to remove some noisy points or blocks, the morphological erosion operation is performed, and two rounds of dilation are conducted to join parts of signs that may be separated due to uneven illumination on the signs. Fig. 4 shows two images taken from our testing images and the corresponding binary images for a warning sign (top) and a prohibitory sign (bottom). The outlines of the road signs are clearly visible in the binary maps, which will be used as candidate regions in the road sign detection stage.

\section{The Detection Module}

The candidate regions obtained from the segmentation stage are verified in this stage according to the symmetric property of their shapes.

In the original RST algorithm [25] and its application on road-sign detection [24], searching was performed on the edges (gradients) across the whole image. Also, gradient elements with small magnitudes were ignored because they had less reliable orientations and were more easily corrupted by noise. This has effectively reduced the searching from the full dimension of the image to a small number of edge pixels which have strong enough contrast to the background. However, searching on certain regions which definitely do not contain a target is unnecessary. Also, for real-life applications which typically have low-resolution targets on cluttered background, such assumptions can easily miss targets. Lastly, this transform suffers from high false alarm rate due to its mono-variate voting scheme.

To reduce the false alarm rate, we propose to use an edge pairwise voting scheme, and name it as IRST algorithm. To improve the efficiency of the searching and the recall rate of the detection, in our algorithm the IRST is performed on the edges of the extracted candidate regions only. This not only avoids unnecessary searching but also helps to retrieve those low-resolution targets and targets with low contrast to background due to motion blur, low illumination, color fading effect, etc.

\subsection{Preparation of Our Algorithm}

In order to illustrate our IRST Algorithm clearly, we first introduce some concepts.

1) The area $S$ of each blob (i.e. the candidate region obtained in Sect. 3) is defined as:

$$
S=\sum_{(x, y) \in R} f(x, y)
$$

where $f(x, y)$ denotes the pixel value of a binary image and $f(x, y)=1$ for pixels belonging to the blob.

2) The roughness of the shape of a blob, denoted as $P$, is defined as:

$$
p=4 \pi S \backslash L^{2},
$$

where $L$ denotes the length of the outer boundary of the blob, and $S$ is the area of the blob. Blobs with different shapes have different $p$ values. The above "area" and "roughness" values are used to estimate the minimum and maximum radius of road signs.

3) The color enhancement image, denoted as $e i$, is obtained as follows:

$$
e i=|| r-g|-| b-y \|,
$$

where $r, g, b$, and $y$ are defined in Eq. 1 .

4) The edge image $e$ is defined as follows:

$$
e=\sqrt{\left(e i \cdot G_{x}\right)^{2}+\left(e i \cdot G_{y}\right)^{2}},
$$


where $G_{x}$ and $G_{y}$ are horizontal and vertical LOG operators respectively.

5) Rotational symmetry is defined as follows: given a pair of non-parallel point vectors $p_{i}$ and $p_{j}$ in general position there exists a point $p$ a distance $r$ from $p_{i}$ and $p_{j}$ about which $p_{i}$ can be rotated to become precisely aligned and coincident with $p_{j}$, denoted as $r c\left(p_{i}, p_{j}\right)=p$. Then, the rotational symmetry centre $p$ is given by:

$$
p=\left(\begin{array}{l}
x_{i} \\
y_{i}
\end{array}\right)+\left(\begin{array}{l}
r \cos (\beta+\gamma) \\
r \sin (\beta+\gamma)
\end{array}\right)
$$

where $x_{i}$ and $y_{i}$ are the Cartesian co-ordinates of $p_{i}, \gamma$ is the angle that the line joining $p_{i}$ and $p_{j}$ makes with the $\mathrm{x}$-axis, and $\beta$ is the angle that the line joining $p_{i}$ and $p_{j}$ makes with the line joining $p_{i}$ and $p$.

\subsection{The Original RST}

In [24], Loy and Barnes presented a method applying the RST to detect road signs with a regular polygon shape (e.g. triangular, square and octagonal road signs) and have achieved fast and reasonably robust algorithm.

For a given pixel $P$, its gradient, denoted as $g$, is calculated using an edge operator that yields orientation, such as Sobel. If this pixel lies on the arc of a circle, then its centre would be in the direction of the gradient, at the distance of the radius. To achieve illumination invariance, they apply the discrete form of the detector. The location of a pixel that will gain a vote as a potential centre is defined as:

$$
P \pm v(P)=A \pm \operatorname{round}\left(r \frac{g(P)}{\|g(P)\|}\right)
$$

where $g(P)$ denotes a unit gradient vector starting at point $P$ and perpendicular to the edge at point $P, r$ is the searching radius, $\|\cdot\|$ is the norm of a vector, and round () rounds each vector element to the nearest integer. There are $P+v(P)$ positively and $P-v(P)$ negatively affected pixels corresponding to points that the gradient points to, at a distance of $r$, and away from $P$ respectively.

At each radius $r$, an orientation projection image $O_{n}$ is generated by examining the gradient ${ }^{2}$ $g$ at each point $P$ from which a corresponding positively affected pixel $P+v(P)$ and negatively affected pixel $P-v(P)$ are determined. A vote image is defined based on these orientation votes as:

$$
O_{P \pm v(P)}=O_{P \pm v(P)} \pm 1 .
$$

Then, the vote image is defined as:

$$
M_{r}=\operatorname{sgn}\left(O_{n}(p)\right)\left(\frac{O_{n}(p)}{k_{n}}\right)^{\sigma}
$$

where $\sigma$ is the radial strictness parameter, and $k_{n}$ is a scaling factor that normalises $O_{n}$ across different radii.

Lastly, vectors projected onto the same pixel are summed. The result is a vector field $B_{r}$, which magnitude indicates how well the gradient elements voting on each point match the target angular spacing. Calculate the output image $S_{r}$ at radius $r$, as $M_{n}(p) B_{r}(p)$, and accommodate for scale. Other details of the RST algorithm can be found in [24]. 


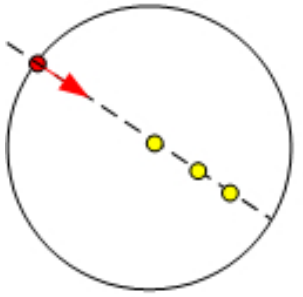

(a)

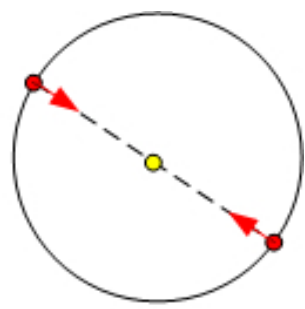

(b)

Fig. 5 Schemes for the voting methods (yellow dots indicate possible centers). (a) The original RST (monovariate voting). (b) Our IRST (pairwise voting)

\subsection{Our Improved RST (IRST)}

The main disadvantage with RST is that it is a mono-variate transformation where any edge point casts its vote in several accumulators independent of its neighbors (as is shown in Fig. 5(a)). This induces a relatively high number of false positives. Moreover, in the original RST algorithm, voting is performed for every edge pixels with strong enough gradient magnitude. No evaluation of the target shape was evaluated in advance. This suffers high computational complexity especially for images with cluttered background such as a city scene. To solve these problems, we propose an improved RST, named as IRST, which helps to detect road signs more efficiently and accurately. Specifically, in order to reduce the high false alarm rate, we propose a voting scheme based on two pixels' positions and orientations (as shown in Fig. 5(b)). Also, in order to reduce the computational complexity, our algorithm searches the image for symmetric features of the expected shapes only.

In our IRST, we uses an edge pairwise voting scheme particularly convenient for reflectional and radial symmetry detection. It is a kind of bi-variate Hough Transform. Therefore it results in fewer false positives and is more noise tolerant than the RST. In our IRST, a pair of gradient vectors with no symmetry may have a significant contribution in the votes for a given point. Our proposed algorithm avoids this impairment of the edge points. We propose here a pairwise voting approach we named as IRST which focuses on pairs of radiant vectors with a strict radial symmetry. The proposed IRST is rather general and can also detect symmetry axis. In this paper, we test our approach for detecting road signs of four shapes (i.e., circle, triangle, rectangle and octagon) in still images. Unlike the original RST, our IRST makes no assumption on light/dark or dark/light contrast of objects to be detected. Our IRST works on intensity images and can detect circular, triangular, square and polygonal shapes regardless of their pose, i.e., it is an orientation free detector, robust to in-plane and small out-of-plane rotations.

Firstly, in Sect. 3, we have obtained candidate regions from the Segmentation module. We then retain the above segmented candidate regions in the edge image $e$, and remove other edges that do not belong to any candidate regions. We denote the resultant new edge map as $\bar{e}$. Our IRST algorithm then operates on the resultant edge image $\bar{e}$ and perform voting for remaining edge pixels. Hence, it reduces the scope of the searching to the utmost extent, which helps to improve the computation efficiency.

Secondly, in our IRST algorithm, we propose to use pairwise voting scheme. For each point $P$ of an image, a set of voters are defined as:

$$
\Gamma(p)=\left\{(i, j) \mid r c\left(p_{i}, p_{j}\right)=p\right\}
$$

The accumulator of the symmetry magnitude at points $P$ is defined as:

$$
\operatorname{Accu}(p)=\sum_{(i, j) \in \Gamma(p)}(C(i, j))
$$

where $C(i, j)$ is the contribution of a pair of points $\left(p_{i}, p_{j}\right)$ to the votes of its middle point $P$. Inspired by the method of intensity in [3]. In our IRST algorithm, $C(i, j)$ is defined as a 
combination of a distance weighting function $D(i, j)$, a phase weighting function $\Phi(i, j)$ and distance $r$ of the pairwise points as:

$$
C(i, j)=D(i, j) \Phi(i, j) r_{i} r_{j},
$$

where $r_{i}$ can be a logarithmic function of the gradient magnitude: $r_{i}=\log \left(1+\left\|n_{i}\right\|\right), n_{i}$ is the gradient vector of $p_{i}$. Due to the pre-determined range of road sign size in the segmentation stage. $D(i, j)$ is represented by a discrete function, which is defined as:

$$
D(i, j)=\left\{\begin{array}{l}
1, i f\left(R_{\min } \leq\left\|p_{i} p_{j}\right\| \leq R_{\max }\right) \\
0, \text { else }
\end{array},\right.
$$

where $R_{\min }$ and $R_{\max }$ are the minimum and maximum width of a road sign respectively, which can be obtained in Sect. 3.

$\Phi(i, j)$ is represented as:

$$
\Phi(i, j)=W\left(\theta_{i}-\alpha_{i j}\right) W\left(\left|\theta_{i}-\theta_{j}\right|-\pi\right),
$$

where $\theta_{i}$ and $\theta_{i}$ denote the gradient orientation of $p_{i}$ and $p_{j}$ respectively, $\alpha_{i j}$ is the angle that the line joining $p_{i}$ and $p_{j}$ makes with the x-axis, and $W$ is a top-hat function, defined as:

$$
W(x)=\left\{\begin{array}{l}
1, \text { if }|x|<\xi \\
0, \text { else }
\end{array}\right.
$$

where $\xi$ is a very small number (in our experiment, it is set as $\frac{\pi}{18}$ ).

Then, we define the scaling factor $k_{N}$ :

$$
k_{N}=\frac{1}{N} \sum_{(i, j) \in \Gamma(p)}\left\|p_{i} p_{j}\right\|
$$

where $N$ is the number of pairwise points.

Lastly, the voting image is defined as:

$$
M_{r}^{\prime}=\operatorname{sgn}(\operatorname{Accu}(p))\left(\frac{A c c u(p)}{k_{N}}\right)^{\sigma},
$$

where $\sigma$ a is the radial strictness parameter.

Regardless of targeting circles or regular polygons, all votes are accumulated into a voting image $M_{r}^{\prime}$, calculated at radius $r$ and accommodating for scale. At the same time, vectors projected onto the same pixel are summed. The result is a vector field $B_{r}^{\prime}$.

Once the voting image $M_{r}^{\prime}$ and the vector field image $B_{r}^{\prime}$ have been computed, the final shape response $S_{r}^{\prime}$ can be determined as:

$$
S_{r}^{\prime}(A)=\frac{M_{r}^{\prime}(A)\left\|B_{r}^{\prime}(A)\right\|}{\left(k_{N} r\right)^{2}} .
$$

Our IRST transform uses the pairwise voting scheme, and therefore reduces the false alarm rate. Also, it is calculated over a set of radii $r \in R$, where $R$ is the set of radii values at which the radius $r$ is determined through analyzing the segmented road sign image. In our experiments, the minimum and maximum radius $r$ of signs are 6 and 60 pixels respectively. In the original RST algorithm, the $r$ is defined by their expectations about the apparent sign size. While in our improved IRST algorithm, it is not necessarily determined in advance and can be obtained by our self-adapted method. For example, when determining radii set $R$, the minimum and maximum radius $r$ are first identified in the segmentation stage by analyzing the candidate regions. Then, whether a radius $r$ falls in the range of [minimum, maximum] is determined. The combined resultant image $S$ is obtained by summing over all $r \in R$. 


\section{(40)金 $\times$}

Fig. 6 Examples of input images and their corresponding voting results.

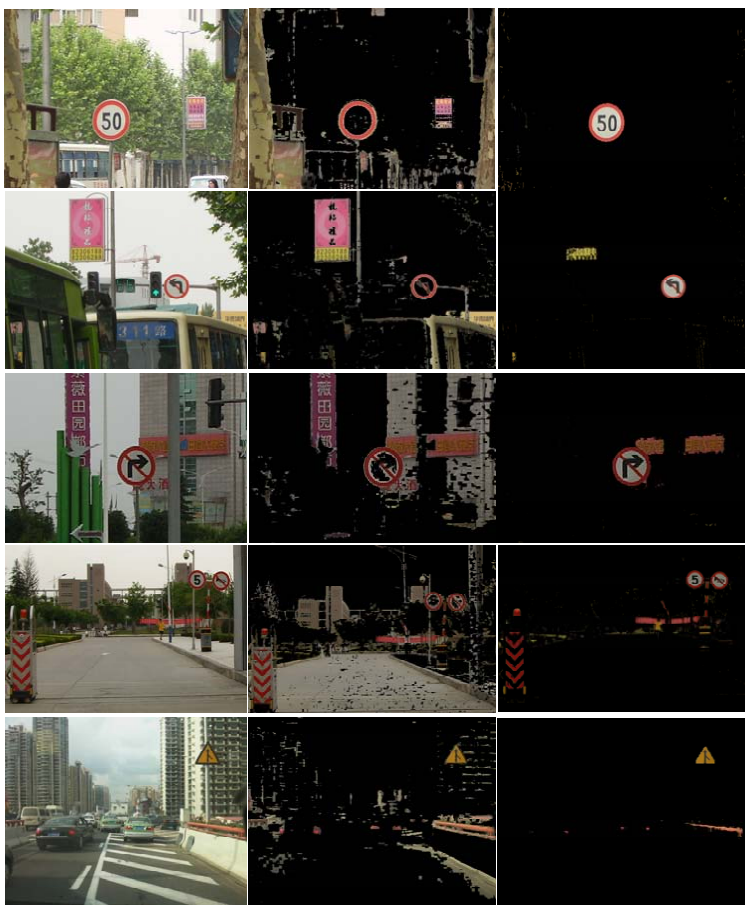

(a)

(b)

(c)

Fig. 7 (a) Original images. (b) Segmentation results using the achromatic color mask of the method in [38]. (c) Segmentation results using our proposed visual saliency algorithm.

To detect the signs, we search the voting image for values above a threshold defined as the required number of pixels for the smallest radius. We then check all the individual radii to find if any are above the threshold of edge pixels for that radius. It could be that a high shape response in Eq. 21 results only from a collection of accidental votes from several contributing radii. If at a particular radius the voting is above the threshold, then this is a possible road sign region. Based on our voting rule, rotational symmetry centers can be detected, as shown in Fig. 6. Thus, our IRST algorithm examines each candidate region in the segmented images and classifies them as either known road signs for output or not. Our proposed algorithm has two output accumulators, one for the center of objects and the other one recording their radius. The center of a target can be extracted from the accumulator. To define the corresponding spatial extent, another accumulator registering the distance between voters is built during the voting process.

\section{Experimental Results and Discussion}

Experimental evaluation was conducted on a large collection of images obtained from the MASTIF Dataset [36], Traffic Signs Dataset [36], German Traffic Sign Recognition Benchmark (GTSRB) Dataset [36], and the rest are captured by us. In this section, we present the experimental evaluation of our proposed road sign detection system. We first present the results on image 
Table 1 Comparison on candidate region generation.

\begin{tabular}{cccc}
\hline Algorithm & Nsr & Ntr & Ndr \\
\hline SMM [38] & 61790 & 3488 & 3401 \\
Ours & $\mathbf{4 3 9 0 8}$ & 3488 & $\mathbf{3 4 2 7}$ \\
\hline
\end{tabular}

segmentation which produces candidate regions for further verification. We then give full quantitative evaluation on the detection accuracy of our proposed algorithm in comparison with the state-of-the-art approaches.

\subsection{Image Segmentation Results}

In order to demonstrate the superiority of our color visual saliency based segmentation algorithm, we compare the performance of our algorithm with that of the saliency map model-based segmentation (SMM) approach in [38] in the Traffic Signs Dataset [36].

Fig. 7 shows some examples of images annotated with the segmentation results generated by our proposed visual saliency algorithm in comparison with those generated using the method presented in [38]. We show the candidate regions in the form of original color images as an intuitive comparison. As it is shown that, using our method (see the third column of Fig. 7), all target signs in the image are accurately partitioned from the background, and some small and big blobs have been removed (blobs having an unrealistic aspect ratio are filtered out in the following processing step before being sent for shape-based verification). This illustrates our color-ratio based visual saliency method can remove more noisy regions which colors appear similar to those of targets.

As a quantitative comparison, the number of segmented regions (Nsr), the number of total road sign regions (Ntr) and the number of detected road sign regions (Ndr) are compared, as shown in Table 1. Clearly, our segmentation algorithm based on visual saliency can detect more road sign regions with much less false alarms for further processing.

\subsection{Road Sign Detection Results}

In order to demonstrate the superior performance of our proposed algorithm, we compare the performance of our algorithm with those of the state-of-the-art approaches on three benchmarking datasets and a newly collected dataset. These approaches include the SVM approach proposed in [26], the approach integrating object detection with 3D tracking in [37], the Fourier descriptors in [21], the ROI extraction and histogram features-based method in [23], the image segmentation and shape analysis method in [20], Mean shift and log-polar transform [8], fast R-CNN method [33] and the RST approach [24]. Detection rate (Hits), false alarm rate (FA) and average processing time are compared. Results are reported per dataset in the next. All experiments were conducted using a computer with $2.19 \mathrm{GHz}$ CPU of 1GB RAM.

\section{Results on the MASTIF Dataset:}

We also implemented the approaches in $[23,21,37]$ and tested them on this dataset. The MASTIF Dataset was acquired with an interlaced camera, and the corresponding signs are often significantly distorted. The dataset contains three datasets (TS2009 (around 6000 signs, cropped sign images), TS2010 (around 3000 signs, full annotated videos), and TS2011 (around 1000 signs, full annotated videos), which we name with respect to the year in which they were annotated. We have therefore used TS2009 for training in most of our experiments so far. For training our detectors we have used TS2009 as is, while our classifiers have been trained on a de-interlaced variant of that dataset. Each sign is annotated $4 \sim 5$ times at different distances from the car. The resolution of the images is $720 \times 576$ pixels. It is publicly available at: http://www.zemris.fer.hr/ ssegvic/mastif/datasets.shtml. 
Table 2 Detection results on the MASTIF Dataset.

\begin{tabular}{cccc}
\hline Algorithm & Hits & FA & Time \\
\hline Ours & $\mathbf{9 6 . 1} \%$ & $\mathbf{0 . 9 5} \%$ & $\mathbf{2 0 0 m s}$ \\
Larsson [21] & $95.1 \%$ & $1.27 \%$ & $2 \mathrm{~s}$ \\
Liang [23] & $95.8 \%$ & $1.03 \%$ & $800 \mathrm{~ms}$ \\
Šegvi [36] & $95 \%$ & $1.28 \%$ & - \\
Timofte [37] & $94.7 \%$ & $1.34 \%$ & $286 \mathrm{~ms}$ \\
Ellahyani [8] & $94.9 \%$ & $1.3 \%$ & $389 \mathrm{~ms}$ \\
Qian [33] & $96 \%$ & $1.27 \%$ & $950 \mathrm{~ms}$
\end{tabular}

Table 3 Detection results on the Traffic Signs Dataset.

\begin{tabular}{cccc}
\hline Algorithm & Hits & FA & Time \\
\hline Ours & $\mathbf{9 6 . 3} \%$ & $\mathbf{0 . 9 7} \%$ & $\mathbf{3 8 5 m s}$ \\
Timofte[37] & $95.6 \%$ & $1.61 \%$ & $3 \mathrm{~s}$ \\
Larsson [21] & $95.4 \%$ & $1.26 \%$ & - \\
Liang [23] & $94.9 \%$ & $1.28 \%$ & $986 \mathrm{~ms}$ \\
Šegvi [36] & $92.9 \%$ & $2.04 \%$ & $451 \mathrm{~ms}$ \\
Ellahyani [8] & $95.1 \%$ & $1.74 \%$ & $419 \mathrm{~ms}$ \\
Qian [33] & $95.8 \%$ & $1.39 \%$ & $1.2 \mathrm{~s}$ \\
\hline
\end{tabular}

Table 2 shows the comparative results obtained on this dataset. As it shows, the results obtained using the method in [23] and [33] are very close to that of our algorithm, but the method in [23] and [33] run at a frame rate of 0.5 frames and 0.95 frames per second respectively, whereas our system runs at a frame rate of approximately 5 frames per second on this dataset. The processing speed in [37] is very close to our method, about $0.2 \sim 0.4$ second per image, but its detection rate is not as comparable as ours. These indicate our algorithm is discriminative and effective.

\section{Results on the Traffic Signs Dataset:}

This dataset contains more than 20000 images with $20 \%$ labeled (including 3488 traffic signs) from highways and cities recorded from more than $350 \mathrm{~km}$ of Swedish roads. A 1.3 mega-pixel color camera, a Point-Grey Chameleon, was placed inside a car on the dashboard looking out of the front window. The camera was pointing slightly to the right, in order to cover as many relevant signs as possible. The lens had a focal length of $6.5 \mathrm{~mm}$, resulting in approximately 41 degrees field of view. Typical speed signs on motorways are about $90 \mathrm{~cm}$ wide, which corresponds to a size of about 50 pixel if they are to be detected at a distance of about $30 \mathrm{~m}$. A human operator started the recording whenever a traffic sign was visible and stopped the recording when no more signs were visible. The label for each sign contains sign type (pedestrian crossing, designated lane right, no standing or parking, priority road, give way, $50 \mathrm{kph}$, or $30 \mathrm{kph}$ ), visibility status (occluded, blurred, or visible) and road status (whether the signs is on the road being traveled or on a side road). The resolution of the images is $1360 \times 800$ pixels. It used a 1.3 mega-pixel color camera, which was placed inside a car on the dashboard looking out of the front window. The dataset is publicly available at: http://www.cvl.isy.liu.se/research/datasets/traffic-signs-dataset.

The results on this dataset, as shown in Table 3, were first reported in [21], which used locally segmented contours combined with an implicit star-shaped model to detect different road signs. However, the performance of its method degrades when there exists low image contrast or various illumination conditions. Note that, the method in [37],[21] and [33] performs comparably with the method in [8], with a slight improvement. Because the dataset contains more mandatory road signs, the performance of the method in [23] drops slightly. Because of low image contrast and various illumination conditions, the unstable performance of temporal and spatial features leads to the poor performance of the method in [36]. Thanks to our color visual saliency based segmentation method, our system can achieve highest detection rate. Moreover, our system runs at a frame rate of approximately 3 frames per second on this dataset. 
Table 4 Detection results on the GTSDB Dataset.

\begin{tabular}{cccc}
\hline Algorithm & Hits & FA & Time \\
\hline Ours & $98.7 \%$ & $0.45 \%$ & $\mathbf{2 5 6 m s}$ \\
Liang [23] & $97 \%$ & $0.51 \%$ & $826 \mathrm{~ms}$ \\
Larsson [21] & $\mathbf{9 9 \%}$ & $\mathbf{0 . 4 3} \%$ & $2.8 \mathrm{~s}$ \\
Khan [20] & $92.7 \%$ & $1.98 \%$ & $40 \mathrm{~s}$ \\
Loy [24] & $96.9 \%$ & $0.88 \%$ & $406 \mathrm{~ms}$ \\
Bascn [26] & $95.8 \%$ & $1.04 \%$ & $498 \mathrm{~ms}$ \\
Timofte [37] & $95.2 \%$ & $1.21 \%$ & $296 \mathrm{~ms}$ \\
Ellahyani [8] & $94.2 \%$ & $1.54 \%$ & $325 \mathrm{~ms}$ \\
Qian [33] & $97.5 \%$ & $0.59 \%$ & $1 \mathrm{~s}$ \\
\hline
\end{tabular}

Table 5 Detection results on the newly collected dataset.

\begin{tabular}{cccc}
\hline Algorithm & Hits & FA & Time \\
\hline Ours & $\mathbf{9 7 . 1} \%$ & $\mathbf{0 . 7 5} \%$ & $\mathbf{3 1 8 m s}$ \\
Larsson [21] & $96.8 \%$ & $0.94 \%$ & $2.4 \mathrm{~s}$ \\
Khan [20] & $92.3 \%$ & $1.95 \%$ & $32 \mathrm{~s}$ \\
Liang [23] & $96.5 \%$ & $1.02 \%$ & $988 \mathrm{~ms}$ \\
Loy [24] & $94.4 \%$ & $0.98 \%$ & $496 \mathrm{~ms}$ \\
Bascn [26] & $93.8 \%$ & $1.24 \%$ & $608 \mathrm{~ms}$ \\
Timofte [37] & $92.6 \%$ & $1.71 \%$ & $351 \mathrm{~ms}$ \\
Ellahyani [8] & $96.1 \%$ & $0.89 \%$ & $408 \mathrm{~ms}$ \\
Qian [33] & $96.7 \%$ & $0.99 \%$ & $1.1 \mathrm{~s}$ \\
\hline
\end{tabular}

\section{Results on the original GTSDB Dataset:}

The GTSDB Dataset is a multi-class, single-image classification challenge dataset. The dataset was created from approximately 10 hours of video that were recorded while driving on different road types in Germany during daytime. The sequences were recorded in March, October and November 2010. For data collection, a Prosilica GC 1380CH camera was used with automatic exposure control and a frame rate of $25 \mathrm{fps}$. The camera images, from which the traffic sign images are extracted, have a resolution of $1360 \times 800$ pixels in PPM format. It is provided for a benchmark at the IEEE International Joint Conference for Neural Networks (IJCNN) 2013. The data is publicly available at: http://benchmark.ini.rub.de/.

Table 4 summarizes the comparative results obtained on this dataset. It can be seen that the results in [33] are very close to our algorithm. This is because the features extracted from CNN are more discriminative. Note that, the method in [23] performs comparably with the method in [24], with a slight improvement. This is because the method has been specially designed for the GTSDB Dataset, with lots of parameters generated from images in this dataset. The method in [8] perform not well, that is because the Mean shift algorithm is not stable in this dataset.

\section{Results on the newly collected dataset:}

We also collected a larger and more diverse dataset which contains in total 1800 images (500 images from the MASTIF Dataset, 500 images from the Traffic Signs Dataset, 300 images from the GTSDB Dataset, and 500 images from our own dataset). The 500 images from our own dataset were collected by us using cameras mounted on moving vehicles, among which 726 are road signs of 42 different types.

Table 5 shows the comparative results of our method and the state-of-the-art methods on the newly collected dataset containing in total 1800 road signs images. The images under testing cover a wide range of environmental conditions, such as sunny, cloudy, and rainy weather; at different times of the day and night; with partial occlusion; cluttered background; and different scaling of the signs. From the results, it can be seen that our proposed technique outperforms all referred approaches in all aspects. 


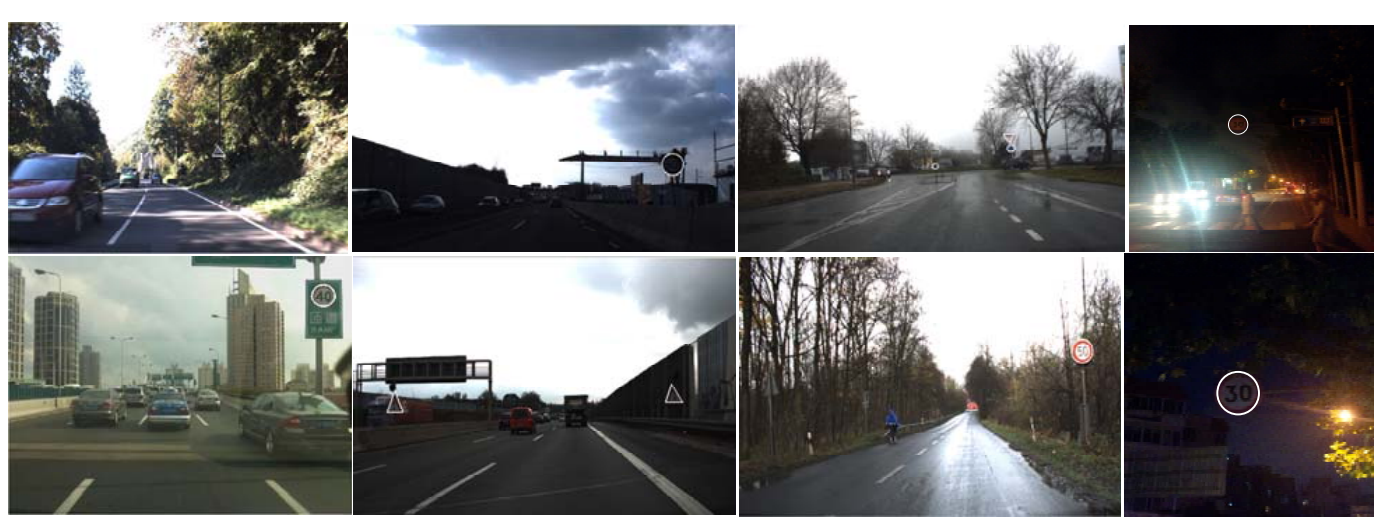

Fig. 8 Examples of detection results when it is sunny (first column), cloudy (second column), rainy (third column) and at night (forth column).
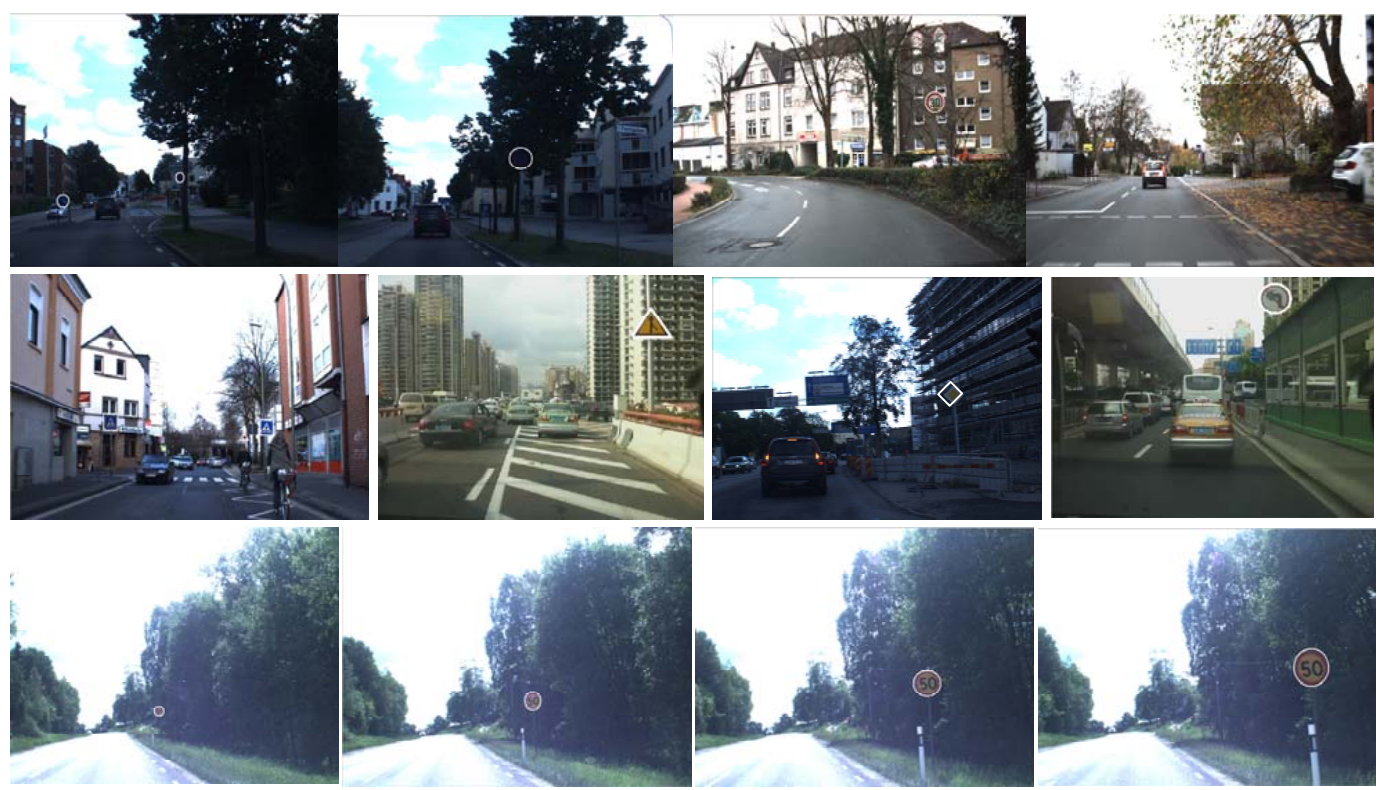

Fig. 9 Examples of detection results with partial occlusion (first row), complex background (second row), and different scaling (third row).

\subsection{Examples of Detection Results}

Examples of detections are shown in Figs.8-9, where the detected road signs are marked in white circles.

Fig. 8 shows examples of road sign detection results in different weather conditions. As is shown, the first column includes the detection results under a sunny day, the second column is the detection results taken when it is cloudy, the third column is the results when it is rainy, and the forth column shows the results at night. We can observe that the results are also encouraging because the material of the road signs can be highly reflective in different weather conditions. Moreover, our system works when arrays of two or more road signs exist in an image.

Fig.9 shows examples of road sign detection with partial occlusion, complex background and different scaling conditions. It is observed that the proposed system can handle mild partial occlusion, uncertain environment, and various scales of road signs. This is because our proposed visual saliency technology is based on biologically motivated selective attention mechanism and imitates human-like early visual processing. To some extent, it addresses the low contrast problems in complex environment. 


\section{Conclusion}

In this paper, a novel algorithm for road sign detection, based on visual saliency and shape analysis, has been proposed. The segmentation-based detection algorithm is found to be robust for its ability to mark a road sign as a candidate region. Then, the shape classification algorithm improves the computation time in the next stage of detection. In general, in this paper we mainly do the following work: in order to solve low contrast problem, segmentation algorithm based on visual saliency is proposed; in order to effectively detect road signs, an IRST algorithm is proposed. The method extends the concept of the fast radial symmetry transform on extracted candidate regions. Experimental results have shown a high detection rate, and the choice of the visual saliency has a profound impact on the performance of detection and recognition.

In future work, we are planning to focus on the following issues: 1) To utilize the developed system for tracking the road signs in a continuous mode from a video sequence. 2) To integrate color information and piecewise affine warping to recover deformation of the road signs in the candidate regions caused by an inappropriate camera view angle, which can reduce the detection and recognition errors.

Acknowledgements This research was partly supported by National Science Foundation, China (No. 61702226, 61672263, 21365008), the Natural Science Foundation of Jiangsu Province (Grant no. BK20170200, BK20161135), the Open Project Program of the Fujian Provincial Key Laboratory of Information Processing and Intelligent Control (Minjiang University, No. MJUKF201709).

\section{References}

1. Aung, A., Fadila, Y., Gondokaryono, Y.: Building robust deep neural networks for road sign detection. arXiv pp. 65-69 (2017)

2. Bar, X., Escalera, S., Vitri, J., Pujol, O., Radeva, P.: Traffic sign recognition using evolutionary adaboost detection and forest-ecoc classification. IEEE Trans. Intell. Transp. Syst. 10(1), 113-126 (2009)

3. Belaroussi, R., Tarel, J.: A real time road sign detection using bilateral chinese transform. Advanced in visual computing pp. 1161-1170 (2009)

4. Benallal, M., Meunier, J.: Real-time color segmentation of road signs. IEEE Can. Conf. Elect. Comput. Eng pp. $1823-1826(2003)$

5. Bouti, A., Mahraz, M.: Robust system for road sign detection and recognition using template matching. Intelligent Systems and Computer Vision pp. 11-19 (2017)

6. Broggi, A., Cerri, P., Medici, P., Porta, P., Ghisio, G.: Real time road signs recognition. IEEE Intell. Vehicles Symp. 34(12), 981-986 (2007)

7. Cheng, H., Jiang, X., Sun, Y., Wang, J.: Color image segmentation: Advances and prospects. Pattern Recognit 34(12), 2259-2281 (2001)

8. Ellahyani, A., Ansari, M.: Mean shift and log-polar transform for road sign detection. Multimedia Tools and Applications pp. 1-19 (2016)

9. Ellahyani, A., Ansari, M.: Mean shift and log-polar transform for road sign detection. Multimedia Tools and Applications pp. 24,495-24,513 (2017)

10. Escalera, A., Armingol, J., Mata, M.: Traffic sign recognition and analysis for intelligent vehicles. Image Vis. Comput. 21(3), 248-257 (2003)

11. Escalera, A., Armingol, J., Pastor, J., Rodriguez, F.: Visual sign information extraction and identification by deformable models for intelligent vehicles. IEEE Trans. on Intel. Trans. Systems 5(2), 57-68 (2004)

12. Escalera, S., Pujol, O., Radeva, P.: Traffic sign recognition system with $\beta$-correction. Machine Vision and Applications 21(2), 99-111 (2010)

13. Gao, X., Podladchikova, L., Shaposhnikov, D., Hong, K., Shevtsova, N.: Recognition of traffic signs based on their colour and shape features extracted using human vision models. J. Vis. Commun. Image Represent $\mathbf{1 7}(4), 675-685$ (2006)

14. Gomes, S., Reboucas, E., Neto, E.: Embedded real-time speed limit sign recognition using image processing and machine learning techniques. Neural Computing and Applications pp. 573-584 (2017)

15. Gomez, L., Fuentes, O.: Color-based road sign detection and tracking. in Proc. Image Analysis and Recognition(ICIAR) pp. 251-257 (2007)

16. Gudigar, A., Chokkadi, S., Acharya, U.: A review on automatic detection and recognition of traffic sign. Multimed.Tools and Appl. 75(1), 333-364 (2016)

17. Gudigar, A., Chokkadi, S., Raghavendraa, U., Acharya, U.: Local texture patterns for traffic sign recognition using higher order spectra. Pattern Recognit.Lett. pp. 1-8 (2017). DOI 10.1016/j.patrec.2017.02.016

18. Itti, L., Koch, C., Niebur, E.: A model of saliency-based visual attention for rapid scene analysis. IEEE Trans. Patt. Mach. Intel 20(11), 1254-1259 (1998)

19. Jun, W., Ha, J., Jeon, B., Lee, J.: Led traffic sign detection with the fast radial symmetric transform and symmetric shape detection. Intelligent Vehicles Symposium. IEEE pp. 310-315 (2015) 
20. Khan, J., Adhami, R., Bhuiyan, S.: Image segmentation and shape analysis for road-sign detection. IEEE Trans. on Intel. Trans. Systems 12(1), 83-96 (2011)

21. Larsson, F., Felsberg, M.: Using fourier descriptors and spatial models for traffic sign recognition. In Proceedings of the 17th Scandinavian Conference on Image Analysis pp. 238-249 (2014)

22. Lee, S., Suhr, J., Jung, H.: Corner detection-based road sign detection for low-cost sensor fusion-based precise localization system. Transactions of The Korean Society of Automotive Engineers pp. 20-31 (2018)

23. Liang, M., Yuan, M., Hu, X., Li, J., Liu, H.: Traffic sign detection by roi extraction and histogram featuresbased recognition. In International Joint Conference on Neural Networks (IJCNN 2013) pp. 1-8 (2013)

24. Loy, G., Barnes, N.: Fast shape-based road sign detection for a driver assistance system. IEEE Conf. Int. Robots Syst pp. 70-75 (2004)

25. Loy, G., Zelinsky, A.: Fast radial symmetry for detecting points of interest. IEEE Trans. Patt. Mach. Intel 25(8), 959-973 (2003)

26. Maldonado-Bascon, S., Lafuente-Arroyo, S., Gil-Jimnez, P., Gomez-Moreno, H., Lopez-Ferreras, F.: Roadsign detection and recognition based on support vector machines. IEEE Trans. Intell. Transp. Syst 8(2), 264-278 (2007)

27. Nguwi, Y., Kouzani, A.: Automatic road sign recognition using neural networks. Proc. Int. Joint Conf. Neural Netw. pp. 3955-3962 (2006)

28. Overett, G., Tychsen-Smith, L., Petersson, L., Pettersson, N., Andersson, L.: Creating robust highthroughput traffic sign detectors using centre-surround hog statistics. Mach.Vision.Appl. 25(3), 713-726 (2014)

29. Paclik, P., Novovicova, J., Duin, R.: Building road-sign classifiers using a trainable similarity measure. IEEE Trans. Intell. Transp. Syst 7(3), 309-321 (2006)

30. Paclik, P., Novovicova, J., Pudil, P., Somol, P.: Road sign classification using laplace kernel classifier. Pattern Recognit.Lett. 12(13), 1165-1173 (2000)

31. Parada-Loira, F., Landesa-Vzquez, I., Alba-Castro, J.: Fast realtime multiclass traffic sign detection based on novel shape and texture descriptors. In: Proceedings of ITSC pp. 1-8 (2010)

32. Park, S., An, K., Lee, M.: Saliency map model with adaptive masking based on independent component analysis. Neurocomputing 49(4), 417-422 (2002)

33. Qian, R., Liu, Q., Yue, Y.: Road surface traffic sign detection with hybrid region proposal and fast r-cnn. International Conference on Natural Computation, Fuzzy Systems and Knowledge Discovery pp. 555-559 (2016)

34. Ruta, A., Li, Y., Vitri, J., Liu, X.: Towards real-time traffic sign recognition by class-specific discriminative features. BMVC pp. 399-408 (2007)

35. Ruta, A., Porikli, F., Watanabe, S., Li, Y.: In-vehicle camera traffic sign detection and recognition. Machine Vision and Applications 22(2), 359-375 (2011)

36. Šegvić, S., Brkić, K., Kalafatić, Z., Pinz, A.: Exploiting temporal and spatial constraints in traffic sign detection from a moving vehicle. Mach.Vision.Appl. 25(3), 649-665 (2014)

37. Timofte, R., Zimmermann, K., Gool, L.: Multi-view traffic sign detection, recognition, and 3d localisation. Mach.Vision.Appl. 25(3), 633-647 (2014)

38. Woong-Jae, M., Minho, L., Joon-Woo, S.: Implementation of road traffic signs detection based on saliency map model. Intelligent Vehicles Symposium pp. 542-547 (2008)

39. Wu, W., Chen, X., Yang, J.: Detection of text on road signs from video. IEEE Trans. on Intel. Trans. Systems 6(4), 378-390 (2005) 